\title{
INITIAL FAR-FIELD OTR IMAGES GENERATED BY 120-GEV PROTONS AT FNAL*
}

\author{
A.H. Lumpkin, Accelerator Institute, Argonne National Laboratory, Argonne, IL 60439 USA \\ V. E. Scarpine, G.R. Tassotto, Fermi National Accelerator Laboratory, Batavia, IL 60510 USA
}

\begin{abstract}
We have successfully imaged for the first time the angular distribution patterns of optical transition radiation (OTR) generated by $120-\mathrm{GeV}$ proton beams passing through an $\mathrm{Al}$ metal plane. These experiments were performed at Fermilab (FNAL) with the same chamber, foil, and camera design as with the near-field experiments previously reported. In this case the lens-to-CID-chip separation was remotely adjusted to provide the focus-atinfinity, or far-field optical imaging. Data have been obtained in transport lines both before the antiproton production target and before the NuMI target with particle intensities of about 5 to $22 \times 10^{12}$. A two-foil interferometer calculation was also performed. Single-foil experimental and modeling results will be presented.
\end{abstract}

\section{INTRODUCTION}

The imaging of the size and profile of intense proton beams at high energy and intensity presents many challenges. These include foil survivability, signal strength, a large clear aperture of the frame, foil flatness and reflectivity, ionizing radiation damage to array sensors and their electronics, and light collection. In the first demonstration of the optical transition radiation (OTR) effect by Goldsmith and Jelley [1], very low energy, but very intense, proton beams were used. The imaging of much higher energy $(14 \mathrm{GeV})$ proton beams was done in the 1980s at CERN in a collaboration with Wartski [2]. More recently a collaboration of ANL and FNAL staff was formed to develop imaging stations for the 120 - to $150-\mathrm{GeV}$ proton beams in several locations of the Fermilab (FNAL) complex. A feasibility study was first reported [3], and then the beam size imaging studies with focus-at-the-object or in the optical near field were reported [4-7]. We now report the first, focus-at-infinity or far-field imaging results on such high-energy intense beams. As is true for the electron or lepton cases [8-10], the far-field images display the angular distribution pattern of the OTR. The general lobe structure for singlefoil emission carries with it dependencies on the complementary properties of beam energy (or gamma), beam pointing angle, and beam divergence. We describe our initial single-foil far-field measurements and modeling results for both single-foil and two-foil interferometric cases.

\footnotetext{
*Work supported by U.S. Department of Energy, Office of Science, Office of Basic Energy Sciences, under Contract No. DE-AC0206CH11357 and by U.S. Department of Energy under Contract No. DE-AC02-CH03000.
}

\section{EXPERIMENTAL BACKGROUND}

The FNAL accelerator complex includes a proton linac, an $8-\mathrm{GeV}$ booster, an 8 - to $150-\mathrm{GeV}$ main injector (MI), and the $1-\mathrm{TeV}$ Tevatron where protons and antiprotons are used in the collider programs for high energy physics. The prototype OTR experiments were done in the AP-1 line in an air gap just upstream (pre-vault area) of the antiproton production target [4]. Beam intensities of $5 \times$ $10^{12}$ were available, and there was reasonable access for experimental adjustments. The system used $12-\mu \mathrm{m}$ Ti or $20-\mu \mathrm{m}$-thick $\mathrm{Al}$ foils oriented at $45^{\circ}$ to the beam direction. The more recent in-vacuum stations have been installed in the Tevatron, the NuMI Transport line before the target, in the AP-1 line again, and in the A150 antiproton transport line. The foils are actually Al-coated pellicles with mylar or Kapton substrates.

The OTR station fabricated at FNAL is shown schematically in Fig. 1 of ref. 7. The large-aperture foil frame is inserted by a rotating mechanism. The stations have dual foils mounted to allow bidirectional beam measurements or use as a reference foil. An aluminized Kapton foil consisting of $0.12 \mu \mathrm{m} \mathrm{Al}$ on $6 \mu \mathrm{m}$ of Kapton was positioned with its surface normal at 45 degrees to the beam direction so that backward OTR was emitted at 90 degrees to the beam direction. The OTR is viewed by an ionizing radiation-resistant charge-injection-device (CID) camera with a lens chosen to provide reasonable FOV for the 85-mm-diameter scene. The camera-to-lens distance can be remotely adjusted to provide near-field or far-field optical focus. The camera is also tilted to provide the Scheimpflug condition for preserving near-field focus across the beam spot as projected on the tilted foil surface. Filter wheels are used to select neutral density filters and the two linear polarizers that sample the orthogonal components of the OTR radial polarization.

\section{ANALYTICAL BACKGROUND}

For completeness some background on OTR is included. Our strategy is to convert particle beam information to visible radiation and then take advantage of imaging technology and image processing programs. OTR is emitted when a charged particle beam transits the interface between different dielectric constants. The effect is a surface phenomenon that can be understood as the collapsing of the electric dipole formed by the approaching beam charge and its image charge in the material at the surface. The radiation is emitted promptly in the order of tens of fs and is broadband including the visible spectrum. Using the formalism from Ref. [10] for coherent optical transition radiation interferometry 
(OTRI) calculations as a starting point, we can still apply it to the incoherent case where the coherence function simplifies to just $N$, the number of particles. The general relationship for the number of photons per unit frequency interval and solid angle is given by a product of several functions in Eq. 1 [10]:

$$
\frac{d^{2} N}{d \omega d \Omega}=\left|r_{\perp, \|}\right|^{2} \frac{d^{2} N_{1}}{d \omega d \Omega} I(\boldsymbol{k}) \mathfrak{I}(\boldsymbol{k}),
$$

where the reflection coefficients $r$ are indicated; the single electron OTR spectral angular distribution is

$$
\frac{d^{2} N_{1}}{d \omega d \Omega}=\frac{e^{2}}{\hbar c} \frac{1}{\pi^{2} \omega} \frac{\left(\theta_{x}^{2}+\theta_{y}^{2}\right)}{\left(\gamma^{-2}+\theta_{x}^{2}+\theta_{y}^{2}\right)^{2}},
$$

where $\theta_{x}$ and $\theta_{y}$ are measured with respect to the angle of specular reflection; $\gamma$ is the Lorentz factor; $I(\boldsymbol{k})$ is the interference term; and $\mathscr{F}(\boldsymbol{k})$ is the coherence function; and $I(\boldsymbol{k})$ is given by

$$
I(\boldsymbol{k})=4 \sin ^{2}\left[\frac{k L}{4}\left(\gamma^{-2}+\theta_{x}^{2}+\theta_{y}^{2}\right)\right],
$$

where $L=$ foil separation and $\boldsymbol{k}$ is the wave vector.

Typically for $\gamma=100$, one photon per $10^{3}$ charged particles is generated in the visible light band over all OTR angles. So, one key to an intense image is, of course, intense particle beams. In recent experiments at the Advanced Photon Source (APS) with electron beams we might have $10^{9}$ particles, but the FNAL complex has beam intensities in the $10^{11}$ to $10^{13}$ range. The other key is to have $\gamma$ large enough so that the peak intensity of emissions at $1 / \gamma$ can allow efficient collection of the radiation cone. We use the backward OTR so the reflection coefficient of the material is involved. In this case "shiny" is better. We observed almost 4 to 5 times more light from $\mathrm{Al}$ foils than the $\mathrm{Ti}$ foils in the prototype experiments. In particular, for our $120-\mathrm{GeV}$ protons we have $\gamma=129$, and the opening angle is about $8 \mathrm{mrad}$. The beam size distribution is seen in the image plane and the far-field angular distribution is seen at the focal plane. By changing the lens-to-CID-sensor separation via remote control, we can select the optical configuration.

For additional sensitivity to beam divergence, the twofoil OTR interferometric (OTRI) technique can be considered. In the case of the proton beams we expect minimal scattering by the first thin foil, so that the beam divergence should be sensed. We used few-cm foil separations and found that the bandwidth needed to be in the $10-\mathrm{nm}$ regime to see the fringe modulation cleanly in the calculations. The model results are given in a later section.

\section{EXPERIMENTAL RESULTS}

The first NuMI OTR images were obtained for 120$\mathrm{GeV}$ protons on June 22, 2006. Subsequently, data were obtained in September 2006 and March 2007. The imaging station is $17 \mathrm{~m}$ upstream of the target and outside of its shield wall, and there were $9.4 \times 10^{12}$ protons per spill. The preliminary results are reported for the nearfield image and the corresponding profiles in Ref. [11]. The observed beam sizes were $\sigma_{\mathrm{x}}=0.94 \mathrm{~mm}$ and $\sigma_{\mathrm{y}}=$ $0.8 \mathrm{~mm}$, which are close to the nominal expected sizes of $1 \mathrm{~mm}$.

By shifting the distance between the lens and the CID sensor, the focus-at-infinity or far-field images were obtained. Using the expected opening angle of $8 \mathrm{mrad}$, the angle calibration was evaluated. The results are shown in Fig. 1 for the total intensity image and with the $\theta_{\mathrm{x}}$ profile shown to the side. By selecting and then inserting the linear polarizers into the light path, we obtained the horizontal and vertical polarization components as shown in Fig. 2 and Fig. 3, respectively. The two lobe structures are not quite the same size in Fig. 2, and there is a hint of a $\theta_{x}-\theta_{y}$ tilt in the two lobes of Fig. 3. In both of the polarized cases the central minimum approaches the baseline, which is indicative of a sub-mrad divergence.
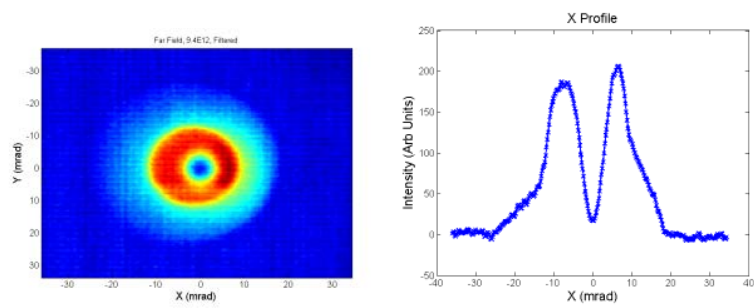

Figure 1: Initial far-field image (left) of OTR angular distribution pattern and horizontal profile (right). A preliminary angle calibration was done based on the lobe peak positions.
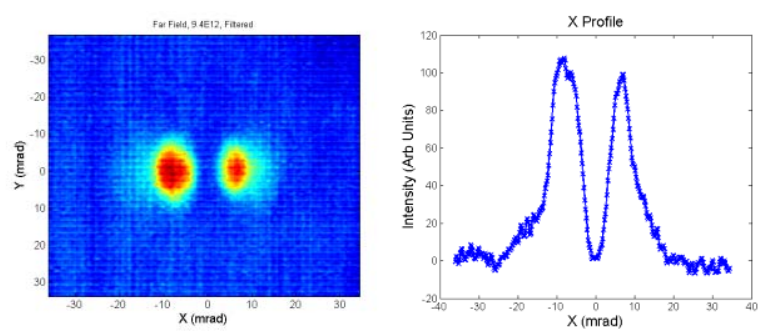

Figure 2: Initial far-field image (left) of OTR and profile (right) of the angular distribution pattern for the horizontal polarization component.
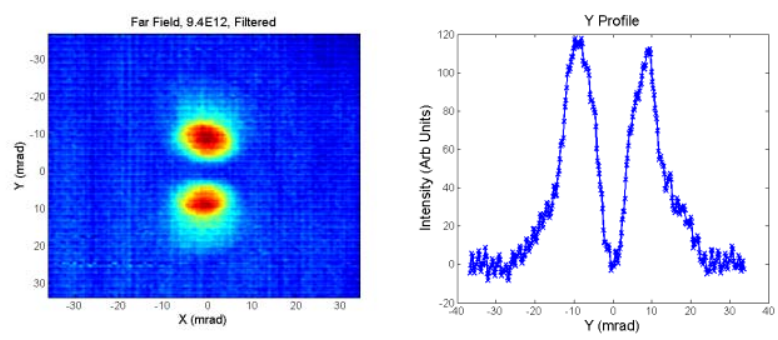

Figure 3: Initial far-field image (left) of OTR and profile (right) of the angular distribution pattern for the vertical polarization component. 
The observed angular distribution patterns indicate the optical alignment is rather good, although the profile shapes are not identical to the theoretical shape. The shape of the profiles suggests some aperturing of the pattern at the 15-mrad level, perhaps by the lens iris. The good alignment means the unexpectedly low photon yield compared to the prototype experiment is probably due to the converter or optics in some way. The position of the pattern provides a relative beam angle monitor at about the 50- $\mu$ rad level. The pattern features are evaluated analytically in the next section.

\section{ANALYTICAL RESULTS}

The single-foil calculations were performed with the analytical code SINGLEF, developed by D.W. Rule a number of years ago [12]. The results of the calculations for the total intensity (black curve) and the two polarization components (Parallel with red dots: perpendicular with green dashes) are shown in Fig. 4 for the 1-mrad divergence case (left). As can be seen the central minimum has approached the baseline in this case in the parallel polarization component. This is typical of looking for beam divergences that are about $1 / 10$ of the opening angle.

To evaluate lower divergence effects, we performed two-foil OTRI calculations using the code GINTERF, also developed by D.W. Rule and ported to the APS computers [13]. We considered the expected divergence for the $95 \%$ emittance of $20 \pi \mathrm{mm} \operatorname{mrad}$ and $\beta_{\mathrm{x}}=40 \mathrm{~m}$. This implies $\sigma_{\mathrm{x}}=0.025 \mathrm{mrad}$. The results of the OTRI calculation are shown in Fig. 4 (right) for the $0.05-\mathrm{mrad}$ case. The spacing was $4 \mathrm{~cm}$, and the bandwidth was 10 $\mathrm{nm}$ at a 500-nm initial wavelength. The fringe modulation depth increases for a lower divergence beam in this regime. This suggests potential sensitivity in the 0.03mrad regime. The beam scattering due to the first thin foil and any transparency issues need further consideration.
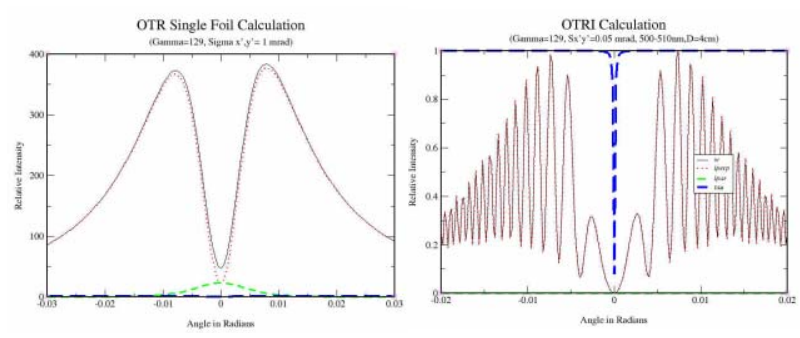

Figure 4: Analytical results for the single-foil case for $1.0 \mathrm{mrad}$ divergence (left) and a two-foil OTRI case for $0.05 \mathrm{mrad}$ divergence (right) with $\gamma=129$. The total intensity (black-solid curve), the parallel polarization component (red-dotted curve), and the perpendicular component (green-dashed curve) are indicated.

\section{SUMMARY}

In summary, we have obtained our first far-field OTR images for 120-protons at the NuMI station. To our knowledge, these are the first such images from an intense, high energy proton beam anywhere. A deep central minimum is observed, which is consistent with a beam divergence below the $0.5-1.0 \mathrm{mrad}$ level as expected. The present light yield limits the far-field images at lower charge intensities. We have also designed a possible OTRI configuration that may be applicable to the antiproton transport line to address the low-divergence beams. Further beam tests would be useful with beam angle changes and focusing adjustments to see the effects on the patterns and to evaluate diagnostic applications.

\section{ACKNOWLEDGMENTS}

The authors acknowledge the support of Kwang-Je Kim (ANL) and Manfred Wendt (FNAL) for our introducing OTR imaging of intense hadron beams to the collider complex.

\section{REFERENCES}

[1] P. Goldsmith and J.V. Jelley, Phil. Mag., Vol. 4, pp. 836-844 (1959).

[2] J. Bosser, J. Mann, G. Ferioli, and L. Wartski, Nucl. Instrum. Methods A 238, 45 (1985).

[3] A.H. Lumpkin and V.E. Scarpine, Proc. of PAC2003, Portland, Oregon, May 2003, 2414-2416 (2003), http://www.jacow.org.

[4] Victor E. Scarpine, Alex H. Lumpkin, Warren Schappert, and Gianni R. Tassotto, IEEE Trans. on Nucl. Sci., 51(4), 1529 (August 2004).

[5] V.E. Scarpine, G.R. Tassotto, and A.H. Lumpkin, Proc. of BIW04, Knoxville, Tennessee, May 3-6, 2004, AIP Conf. Proc. 732, 413 (2004).

[6] A.H. Lumpkin, V.E. Scarpine, Proc. of AAC04, AIP Conf. Proc. 737, 359 (2004).

[7] V.E. Scarpine, C.W. Lindenmeyer, G.R. Tassotto, and A.H. Lumpkin, Proc. of PAC2005, Knoxville, TN, June 2005, 2381-2383 (2005), http://www.jacow. org.

[8] L. Wartski, J. Marcow, and S. Roland, IEEE Trans. Nucl. Sci., 20, 544-548 (1973).

[9] R.B. Fiorito and D.W. Rule, AIP Conf. Proc. 319 (Robert E. Shafer, ed., Santa Fe, NM) pp. 21-37 (1993).

[10]D.W. Rule and A.H. Lumpkin, Proc. of the PAC2001, Chicago, IL, June 2001, 1288-1290 (2001), http://www.jacow.org.

[11] V.E. Scarpine, G.R. Tassotto, and A.H. Lumpkin, "OTR Imaging of Intense $120 \mathrm{GeV}$ Protons in the NuMI Beamline at FNAL," these proceedings.

[12]D.W. Rule, Code SINGLEF, transferred to APS UNIX system in 1993.

[13]D.W. Rule, Code GINTERF, transferred to APS UNIX system in 1993. 